\title{
Persistência de endossulfam e seu metabólito sulfato de endossulfam em condições de campo e laboratório
}

\author{
Rômulo P. Scorza Júnior ${ }^{1}$, André A. Franco ${ }^{1}$ \& Leila C. K. Moraes ${ }^{2}$
}

\begin{abstract}
RESUMO
Objetivou-se, neste trabalho, avaliar a persistência e a lixiviação de endossulfam no campo e sua degradação e formação do metabólito sulfato de endossulfam em condições de laboratório. Visando ao experimento da persistência e à lixiviação no campo, amostras de solo até a profundidade de $100 \mathrm{~cm}$ foram coletadas aos 0, 41, 74 e 125 dias após aplicação. Em laboratório amostras de solo nas profundidades de 0-30 e 50-70 $\mathrm{cm}$ foram incubadas a $30 \pm 2{ }^{\circ} \mathrm{C}$ e $80 \%$ da capacidade de campo e os resíduos de endossulfam e sulfato de endossulfam foram quantificados em função do tempo para ajuste dos modelos de cinética de primeira ordem e do modelo bifásico. Observou-se, no campo, que o endossulfam não lixiviou para menos de 10 $\mathrm{cm}$ de profundidade e houve uma rápida dissipação resultando em uma quantidade remanescente no perfil do solo $(100 \mathrm{~cm})$ inferior a $1 \%$ da dose aplicada aos 41 dias após a aplicação. Em laboratório os valores de $\mathrm{TD}_{50}$ para o endossulfam variaram de 17 a 26 dias e para o sulfato de endossulfam de 26 a 59 dias. $\mathrm{O}$ modelo de degradação bifásico mostrou-se superior ao de cinética de primeira ordem para descrever a degradação do endossulfam em laboratório.
\end{abstract}

Palavras-chave: degradação, meia-vida, lixiviação, dissipação

\section{Persistence of endosulfan and its metabolite endosulfan sulfate under field and laboratory conditions}

\begin{abstract}
This study aimed to evaluate endosulfan leaching and persistence in the field as well as its degradation and formation of the metabolite endosulfan sulfate under laboratory conditions. For the persistence experiment and leaching in the field, soil samples until $100 \mathrm{~cm}$ depth were collected at $0,41,74$, and 125 days after application. In the laboratory, soil samples of $0-30 \mathrm{~cm}$ and $50-70 \mathrm{~cm}$ depth were incubated at $30 \pm 2{ }^{\circ} \mathrm{C}$ and $80 \%$ field capacity and endosulfan residues were quantified as a function of time for adjusting the first order kinetics and biphasic models. In the field, it was observed that endosulfan did not leach beyond $10 \mathrm{~cm}$ depth and there was a rapid dissipation resulting in a quantity in the soil profile $(100 \mathrm{~cm})$ less than $1 \%$ of the applied dose at 41 days after application. In laboratory TD $_{50}$ values for endosulfan ranged from 17 to 26 days and endosulfan sulfate 26-59 days. The biphasic decay model was superior to the first-order kinetics to describe the degradation of endosulfan in the laboratory.
\end{abstract}

Key words: degradation, half-life, leaching, dissipation 


\section{INTRODUÇÃO}

Informações sobre o comportamento ambiental de agrotóxicos são de fundamental importância para avaliar sua periculosidade ambiental e, desta forma, o potencial de contaminação dos recursos naturais. Os principais processos relacionados ao comportamento ambiental de agrotóxicos são a lixiviação, escoamento superficial, volatilização, sorção e degradação (Köhne et al., 2009). A degradação é relevante para atenuação dos níveis de resíduos de um agrotóxico no solo e se refere ao seu processo de transformação em moléculas menores, de forma biótica ou abiótica tornando-os, na maioria dos casos, moléculas menos tóxicas (Oliveira \& Brighenti, 2011).

A persistência de um agrotóxico no solo é representada pelo seu valor de meia-vida $\left(\mathrm{TD}_{50}\right)$ que corresponde ao tempo necessário, em dias, para degradação de $50 \%$ da dose inicial aplicada. Quanto menor o valor de $\mathrm{TD}_{50}$ mais rápida é a degradação de um agrotóxico e, portanto, menor sua persistência. Os valores de $\mathrm{TD}_{50}$ são estimados com base em modelos matemáticos que descrevem a cinética de degradação de agrotóxicos, sendo o de cinética de primeira ordem o mais utilizado (Aldworth \& Jackson, 2008). No entanto, alguns agrotóxicos têm apresentado comportamento de degradação do tipo bifásico, caracterizado por uma fase mais rápida seguida de uma mais lenta. Diversos modelos matemáticos têm sido utilizados para descrever a degradação bifásica (Gustafson \& Holden, 1990; Guidance, 2010). As principais razões para comportamento bifásico da degradação de agrotóxicos em solos, são a diminuição da sua concentração na fase líquida ao longo do tempo devido aos processos de sorção e difusão em condições de não equilíbrio (Pignatello, 2000), diminuição da atividade microbiana nos solos incubados ao longo do tempo em razão da disponibilidade limitada de fontes de carbono e nutrientes e variabilidade espacial em nível microscópico das taxas de degradação (Gustafson \& Holden, 1990). O valor de $\mathrm{TD}_{50}$ é utilizado para classificação da periculosidade ambiental de um agrotóxico. Assim, sua estimativa deve ser feita de forma bastante criteriosa e fundamentada, o que exige a escolha adequada e correta do modelo matemático a ser utilizado para descrever a cinética de degradação.

O endossulfam (1,4,5,6,7,7-hexachloro-8,9,10-trinorborn5-en-2,3-ylenebis methylene) sulfite é um inseticida acaricida do grupo do organoclorados, utilizado para o controle de pragas nas culturas de soja, algodão, café e cana-de-açúcar, por meio de pulverização sobre as folhas ou incorporação ao solo. O principal metabólito do endossulfam no solo após sofrer oxidação microbiológica, é o sulfato de endossulfam (Kennedy et al., 2001), que é altamente tóxico aos organismos aquáticos (Leonard et al., 2001) além de apresentar maior toxicidade aos seres humanos que o próprio endossulfam. Diante disto, o governo brasileiro determinou a proibição de seu uso a partir de 31 de julho de 2013 (ANVISA, 2010).

Desta forma, objetivou-se: i) avaliar a lixiviação e a persistência do endossulfam em um Latossolo Vermelho distrófico em condições de campo em Ponta Porã, MS, tal como sua degradação em condições de laboratório; ii) avaliar a cinética de formação e a degradação do metabólito sulfato de endossulfam em condições de laboratório e iii) avaliar diferentes modelos matemáticos para descrever a cinética de degradação do endossulfam e a formação/degradação do metabólito sulfato de endossulfam.

\section{Material e Métodos}

O experimento de lixiviação e persistência do endossulfam no campo foi instalado na Fazenda Experimental da Embrapa Agropecuária Oeste em Ponta Porã, MS (22 33 ' 13,4” S; $55^{\circ} 38^{\prime} 53,3^{\prime \prime} \mathrm{O}$ ), em uma área de $900 \mathrm{~m}^{2}$. O solo da área é classificado como Latossolo Vermelho distrófico típico de textura média (Santos et al., 2006). O período experimental compreendeu de 19/12/2008 a 23/04/2009. A aplicação do endossulfam foi realizada no dia 19/12/2008 utilizando-se um pulverizador acoplado ao trator com barra de $14 \mathrm{~m}$ e bicos do tipo leque, espaçados $50 \mathrm{~cm}$ com vazão de $320 \mathrm{~L} \mathrm{ha}^{-1}$. A dose aplicada de endossulfam foi de $350 \mathrm{~g}$ i.a. $\mathrm{ha}^{-1}$, recomendada para controle de percevejos na cultura da soja. Amostras de solo para quantificação dos resíduos de endossulfam foram coletadas nas profundidades de $0-10 \mathrm{~cm}, 10-30 \mathrm{~cm}, 50-70 \mathrm{~cm}$ e $70-100 \mathrm{~cm}$ aos $0(19 / 12 / 2008), 41(29 / 01 / 2009), 74(03 / 03 / 2009)$ e 125 (23/04/2009) dias após aplicação. Em cada data de amostragem foram sorteadas quatro parcelas de $9 \mathrm{~m}^{2}$, representando cada quadrante da área experimental, para a coleta das amostras de aproximadamente $200 \mathrm{~g}$ as quais foram armazenadas em freezer a $-20^{\circ} \mathrm{C}$ durante 25 dias até a análise.

A metodologia de extração dos resíduos de endossulfam total, que correspondeu ao somatório dos isômeros $\alpha$ e $\beta$ e do seu metabólito sulfato de endossulfam no solo, foram adaptadas de Corrêa et al. (2003). Para a extração $50 \mathrm{~g}$ de solo foram pesados em um erlenmeyer de $500 \mathrm{~mL}$, com tampa, no qual se adicionaram $150 \mathrm{~mL}$ de acetona, mantendo-os sob agitação por uma hora; em seguida, os erlenmeyers foram deixados em repouso durante duas horas, para decantação. Uma alíquota de $6 \mathrm{~mL}$ do sobrenadante foi retirada e transferida para um balão volumétrico e só então passada em um funil contendo $\mathrm{Na}_{2} \mathrm{SO}_{4}$ para retenção de água; posteriormente, toda a acetona foi evaporada através de rotaevaporador e os resíduos (endossulfam e sulfato de endossulfam) presentes no balão foram transferidos para colunas de sílica gel $60(1,5 \mathrm{~cm}$ de diâmetro interno, 8 $\mathrm{cm}$ de comprimento e $1,0 \mathrm{~g}$ de sílica gel) com o objetivo de purificação do extrato; para isto, os resíduos foram dissolvidos em hexano e transferidos para a coluna em três lavagens sucessivas de $5 \mathrm{~mL}$. A eluição do endossulfam e seu metabólito sulfato de endossulfam na coluna de sílica foram feitos utilizando-se a mistura hexano:acetato de etila (7:3), por meio de duas lavagens com $5 \mathrm{~mL}$. Todo o eluado foi evaporado em rotaevaporador e elevado para volume final de $4 \mathrm{~mL}$ em acetona. Todas as amostras foram filtradas em filtro $0,45 \mu \mathrm{m}$ antes da injeção no cromatógrafo. As quantificações do endossulfam e seu metabólito sulfato de endossulfam foram feitos em um cromatógrafo gasoso Varian (modelo CP 3800) operando com detector de captura de elétrons, coluna CP-SIL 5 CB-MS (30 m x $0,25 \mathrm{~mm}$ x $0,25 \mu \mathrm{m} 100 \%$ dimetilpolisiloxano) e amostrador automático. As condições de operação do cromatógrafo foram: fluxos do gás de arraste $\left(\mathrm{N}_{2}\right)$ de $1 \mathrm{~mL} \mathrm{~min}^{-1} \mathrm{e}$ "make-up" de 30 $\mathrm{mL} \mathrm{min}^{-1}$; temperatura de 220 e $320^{\circ} \mathrm{C}$, respectivamente, para injetor e detector; temperaturas da coluna programadas para 100 
${ }^{\circ} \mathrm{C}(1 \mathrm{~min})$, rampa de $10^{\circ} \mathrm{C} \mathrm{min}^{-1}$ até $150^{\circ} \mathrm{C}$ mantida por $1 \mathrm{~min}$, rampa de $13{ }^{\circ} \mathrm{C} \mathrm{min}^{-1}$ mantida durante $1 \mathrm{~min}$, rampa de $15^{\circ} \mathrm{C}$ $\min ^{-1}$ até $200{ }^{\circ} \mathrm{C}$ mantida por $1 \mathrm{~min}$, rampa de $20{ }^{\circ} \mathrm{C}$ min $^{-1}$ até $230^{\circ} \mathrm{C}$ mantida por $1 \mathrm{~min}$, rampa de $25^{\circ} \mathrm{C} \mathrm{m^{-1 }}$ até $260{ }^{\circ} \mathrm{C}$, mantida por $1 \mathrm{~min}$, rampa de $30^{\circ} \mathrm{C} \mathrm{min}$ min $^{-1}$ até $280^{\circ} \mathrm{C}$ mantida por $1 \mathrm{~min}$, rampa de $30{ }^{\circ} \mathrm{C} \mathrm{m^{-1 }}$ até $300^{\circ} \mathrm{C}$ mantida por $0,5 \mathrm{~min} \mathrm{e}$ modo de injeção do tipo "split/splitless". Os padrões analíticos do endossulfam alfa, beta e sulfato de endossulfam foram adquiridos da AccuStandard ${ }^{\circledR}$ e tinham pureza de 99,7, 97,5 e $99,3 \%$, respectivamente. Prepararam-se as curvas analíticas utilizando-se seis concentrações no intervalo de 0,001 a 0,1 $\mu \mathrm{g} \mathrm{mL}^{-1}$ e se obtendo valores de $\mathrm{R}^{2}$ entre 0,9895 a 0,9916 . Para os testes de eficiência dos procedimentos analíticos para extração e quantificação dos resíduos de endossulfam $\alpha, \beta$ e sulfato de endossulfam utilizaram-se três níveis de fortificação $\left(0,02 ; 0,1\right.$ e $\left.2 \mu \mathrm{g} \mathrm{g}^{-1}\right)$ em triplicata, obtendo-se recuperações de 99,101 e $115 \%$, respectivamente, com coeficientes de variação entre 2,3 e 7,4\%. O limite de quantificação de endossulfam total e de sulfato de endossulfam foi de $0,002 \mu \mathrm{g} \mathrm{g}^{-1}$ de solo.

Para o experimento de degradação do endossulfam e formação do metabólito sulfato de endossulfam em condições de laboratório, oito amostras de solo foram coletadas nas profundidades de 0-30 e 50-70 cm, ao acaso, em área vizinha à área onde se realizou o experimento de lixiviação e a persistência no campo. Após a coleta as amostras correspondentes a cada profundidade foram misturadas e homogeneizadas e em seguida secadas ao ar e na sombra, trituradas em moinho e peneiradas em malha de $2 \mathrm{~mm}$. Os valores de $\mathrm{pH}$ (água), matéria orgânica $\left(\mathrm{g} \mathrm{kg}^{-1}\right)$, areia ( $\mathrm{g}$ $\left.\mathrm{kg}^{-1}\right)$ e argila $\left(\mathrm{g} \mathrm{kg}^{-1}\right)$ foram de 5,$2 ; 16 ; 762$ e 194 para $0-30$ $\mathrm{cm}$ e de 5,$0 ; 8 ; 729$ e 244 para $50-70 \mathrm{~cm}$ de profundidade, respectivamente. Para incubação $50 \mathrm{~g}$ de solo seco foram pesados e adicionados em copos de vidro $(200 \mathrm{~mL})$. Alíquotas $(30 \mu \mathrm{L})$ da solução de trabalho de endossulfam total foram adicionadas separadamente ao solo, em cada copo, de modo a fornecer $30 \mu \mathrm{g}$ de endossulfam total em cada copo; esta quantidade corresponde à dose recomendada para controle de percevejos na cultura da soja; no total, 36 copos foram preparados, correspondentes a três repetições para cada data de amostragem $(0,11,19,29,43$ e 63 dias após aplicação) em cada profundidade. Os copos foram fechados na parte superior com papel alumínio contendo seis furos com vista a manter a condição aeróbica. As amostras foram incubadas em incubadoras tipo BOD com temperatura controlada de $30 \pm 2{ }^{\circ} \mathrm{C}$, na ausência de luz e umidade correspondente a $80 \%$ da capacidade de campo (CC). Os valores de umidade volumétrica correspondentes a $80 \%$ da $\mathrm{CC}$ para $0-30 \mathrm{~cm} \mathrm{e}$ $50-70 \mathrm{~cm}$ de profundidade foram, respectivamente, de $0,23 \mathrm{e}$ $0,22 \mathrm{~cm}^{3} \mathrm{~cm}^{-3}$. A umidade nas amostras foi mantida em $80 \%$ da CC por meio da adição periódica de água ultrapura com auxílio de uma balança até atingir o peso desejado em cada copo. A metodologia de extração e a quantificação dos resíduos de endossulfam e sulfato de endossulfam foram semelhantes às descritas anteriormente.

Em função do tempo e para cada profundidade, os valores das quantidades remanescentes de endossulfam total foram ajustados ao modelo de cinética de primeira ordem e ao modelo bifásico (Gustafson \& Holden, 1990). Para tal, utilizou-se o programa Sigma Plot ${ }^{\circledR}$ versão 12.2 pelo método do ajuste de regressão não linear. $\mathrm{O}$ modelo de cinética de primeira ordem é dado pela Eq. 1 .

$$
\mathrm{C}=\mathrm{C}_{0} \mathrm{e}^{-\mathrm{kt}}
$$

em que:

C - quantidade remanescente de endossulfam total no solo, $\%$

$\mathrm{C}_{0}$ - quantidade inicial aplicada, \%

k - taxa de degradação do endossulfam total, $\mathrm{d}^{-1}$

t $\quad$ - tempo de incubação, dias

O modelo bifásico é dado pela Eq. 2 .

$$
\mathrm{C}=\mathrm{C}_{0}(1+\beta \mathrm{t})^{-\alpha}
$$

em que:

$\alpha \quad$ - parâmetro adimensional

$\beta \quad$ - parâmetro, $\mathrm{d}^{-1}$

Em função do tempo no solo, os dados da quantidade do metabólito sulfato de endossulfam (cinética de formação e degradação) foram ajustados ao modelo de cinética de primeira ordem (Richter et al., 1996) dado pela Eq. 3:

$$
\mathrm{C}_{\text {sul }}=\frac{\mathrm{C}_{0 \_ \text {sul }} \mathrm{k}_{\text {sul }}}{\mathrm{k}-\mathrm{k}_{\mathrm{sul}}}\left[\mathrm{e}^{-\mathrm{k}_{\text {sul }} \mathrm{t}}-\mathrm{e}^{-\mathrm{kt}}\right]
$$

em que:

$\mathrm{C}_{\text {sul }}$ - concentração de sulfato de endossulfam no solo, $\mu \mathrm{g} \mathrm{g}^{-1}$

$\mathrm{C}_{0 \_ \text {sul }}$ - concentração inicial de sulfato de endossulfam no solo, $\mu \mathrm{g} \mathrm{g} \mathrm{g}^{-1}$

$\mathrm{k}_{\text {sul }} \quad$ - taxa de degradação de sulfato de endossulfam, $\mathrm{d}^{-1}$

Para o ajuste dos dados da quantidade do metabólito sulfato de endossulfam em função do tempo no solo, utilizou-se o programa KinGUI 2 (Meyer, 2012).

A qualidade do ajuste dos dados observados aos dois modelos foi realizada com base na análise visual, no erro do teste chi-quadrado - Erro $\chi^{2}$ (Guidance, 2010), na significância dos parâmetros pelo teste t a $5 \%$ e na análise dos resíduos padronizados (Neter et al., 1996). O teste chi-quadrado indica se o modelo é apropriado para descrever os dados observados testando se as diferenças entre os valores observados e os preditos são devidas ao acaso. Ante a possibilidade dos dois modelos testados serem considerados apropriados pelo teste chi-quadrado, utilizou-se o Erro $\chi^{2}$ sendo que, quanto menor seu valor menor também será a incerteza assumida pelo modelo nos valores observados considerando-se que tenha passado pelo teste chi-quadrado. Assim, o modelo que apresentar menor valor do Erro $\chi^{2}$ é considerado mais robusto para descrever os dados observados (Guidance..., 2010).

Os valores de $\mathrm{TD}_{50}$ e do tempo para degradação de $90 \%$ da dose inicial aplicada $\left(\mathrm{TD}_{90}\right)$ para o endossulfam e sulfato de endossulfam, após ajuste ao modelo de cinética de primeira ordem, foram obtidos pelas Eqs. 4 e 5, respectivamente. 


$$
\begin{aligned}
& \mathrm{TD}_{50}=\frac{0,693}{\mathrm{k}} \text { ou } \frac{0,693}{\mathrm{k}_{\text {sul }}} \\
& \mathrm{TD}_{90}=\frac{2,303}{\mathrm{k}} \text { ou } \frac{2,303}{\mathrm{k}_{\text {sul }}}
\end{aligned}
$$

Já os valores de $\mathrm{TD}_{50} \mathrm{e} \mathrm{TD}_{90}$ para o endossulfam, após ajuste ao modelo bifásico foram obtidos por:

$$
\begin{aligned}
& \mathrm{TD}_{50}=\frac{1}{\beta}\left(2^{1 / \alpha}-1\right) \\
& \mathrm{TD}_{90}=\frac{1}{\beta}\left(10^{1 / \alpha}-1\right)
\end{aligned}
$$

\section{Resultados e Discussão}

A quantidade média recuperada de endossulfam total logo após aplicação no campo foi, em 19/12/2008, de 1.095,6 $\pm 120,6$ $\mu \mathrm{g} \mathrm{kg}^{-1}$ de solo, indicando uma recuperação de $94 \%$ (baseada na quantidade aplicada de $1.166 \mu \mathrm{g} \mathrm{kg}^{-1}$ ). Observou-se, com base nas amostragens aos 41, 74 e 125 dias após aplicação, que as concentrações de endossulfam na camada de $0-10 \mathrm{~cm}$ de profundidade, foram de 8,85 $\pm 4,12 ; 4,31 \pm 2,00$ e 9,24 $\pm 6,48 \mu \mathrm{g}$ $\mathrm{kg}^{-1}$ de solo, respectivamente. $\mathrm{O}$ endossulfam não foi observado nas camadas abaixo de $10 \mathrm{~cm}$ de profundidade indicando sua baixa lixiviação para as condições edafoclimáticas estudadas. Essas quantidades presentes no solo aos 41, 74 e 125 dias após aplicação representam menos de $1 \%$ da quantidade aplicada evidenciando a rápida dissipação do endossulfam no campo. Na última data de amostragem (125 dias), observouse um valor da quantidade remanescente de endossulfam total no solo $\left(9,24 \mu \mathrm{g} \mathrm{kg}^{-1}\right)$ superior ao das datas anteriores; no entanto, esperava-se um decréscimo da concentração total em função do tempo; esta discrepância se deve, possivelmente, à variabilidade espacial nas concentrações de endossulfam no solo pois se espera que este efeito seja mais pronunciado para as concentrações menores. Corrêa et al. (2003) observaram, em condições de campo em Piracicaba, SP, que o endossulfam não lixiviou abaixo de $20 \mathrm{~cm}$ de profundidade e atribuíram este fato às características lipofílicas do endossulfam $\left(\mathrm{K}_{\mathrm{OC}}>5700\right.$ $\mathrm{mL} \mathrm{g}^{-1}$ ), o que proporciona sua alta afinidade pelas partículas do solo. Da mesma forma, Laabs et al. (2000) observaram que o $\alpha$-endossulfam ficou imóvel no solo após aplicação em uma área agrícola de Mato Grosso.

A rápida dissipação do endossulfam no campo observada neste estudo se deveu, possivelmente, à sua volatilização e ao escoamento superficial nos primeiros dias após a aplicação. Nos primeiros 41 dias após a aplicação o endossulfam se concentrava na camada superficial do solo e a temperatura média máxima do ar observada neste período foi de $32{ }^{\circ} \mathrm{C}$ com precipitação total igual a $233 \mathrm{~mm}$. Estudos têm mostrado evidências de volatilização intensa de agrotóxicos em condições tropicais brasileiras (Laabs et al., 2000; Nogueira et al., 2012; Scorza Júnior \& Regitano, 2012). Laabs et al. (2000) observaram $\mathrm{TD}_{50}$ para $\alpha$-endossulfam igual a 2,5 dias após sua aplicação em condições de campo em Mato Grosso. Este pequeno valor de $\mathrm{TD}_{50}$ indica uma rápida dissipação do $\alpha$-endossulfam logo após a aplicação que, provavelmente, foi causada por sua rápida volatilização em virtude das altas temperaturas da região. A presença de resíduos de agrotóxicos em amostras de água da chuva tem sido utilizada para demonstrar a volatilização desses compostos em países de clima temperado (Dubus et al., 2000; Vogel et al., 2008; Gish et al., 2011) e tropical (Laabs et al., 2002; Nogueira et al., 2012), inclusive com relatos da presença do endossulfam (Scheyer et al., 2005; Nogueira et al., 2012). Kennedy et al. (2001) observaram volatilização de $70 \%$ do endossulfam após sua aplicação na cultura do algodoeiro, na Austrália.

Observa-se um comportamento de degradação similar do endossulfam para ambas as profundidades, não evidenciando grande influência da diferença dos atributos físicos e químicos das diferentes profundidades (Figura 1). O ajuste das quantidades remanescentes de endossulfam em função do tempo ao modelo de cinética de primeira ordem foi satisfatório, resultando em baixos valores de Erro $\chi^{2}$ e iguais a 6,3 para $0-30$ $\mathrm{cm}$ e 7,6 para $50-70 \mathrm{~cm}$ de profundidade (Tabela 1). Entretanto, o ajuste ao modelo bifásico de Gustafson \& Holden (1990) resultou em valores de Erro $\chi^{2}$ iguais a 2,6 para $0-30 \mathrm{~cm} \mathrm{e}$ 7,0 para $50-70 \mathrm{~cm}$ de profundidade, que são inferiores aos obtidos para o modelo de cinética de primeira ordem (Tabela 2 ). Esses valores inferiores de Erro $\chi^{2}$ para o modelo bifásico indicam sua superioridade quando comparado com o modelo

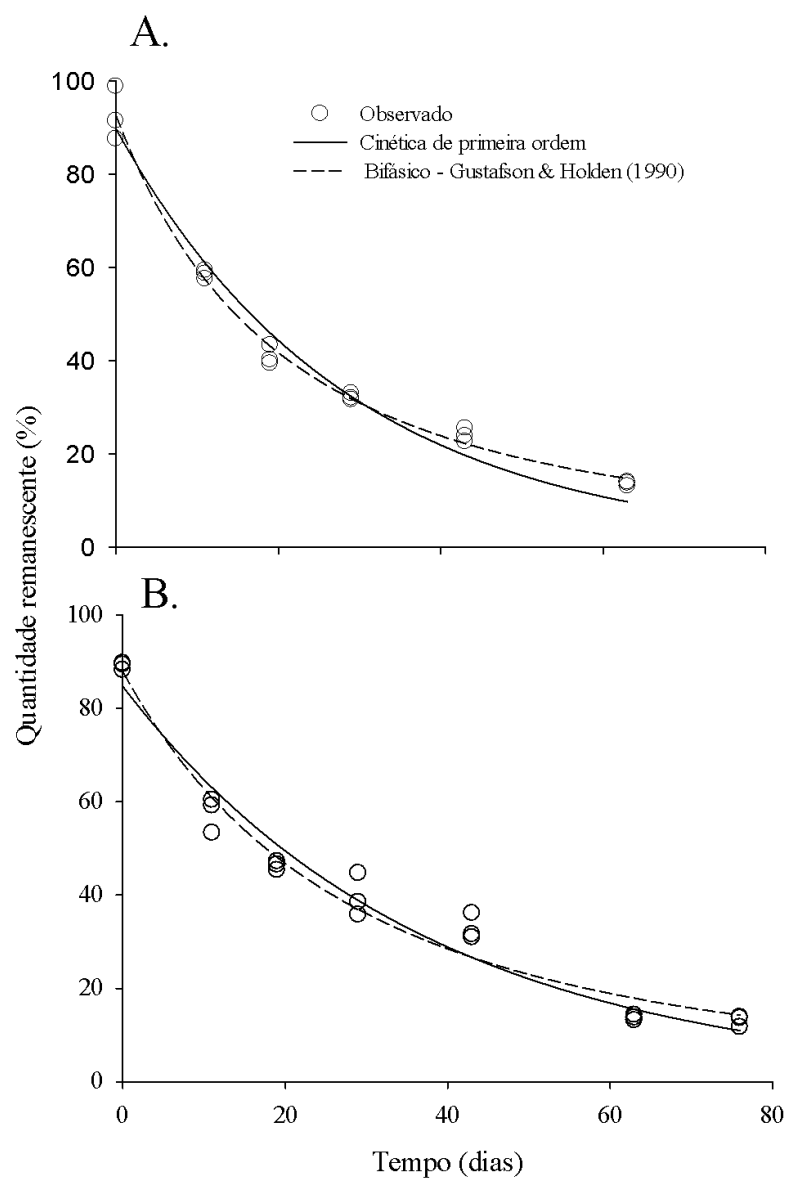

Figura 1. Degradação do endossulfam em um Latossolo Vermelho distrófico em Ponta Porã, MS, incubado a $30{ }^{\circ} \mathrm{C}$, umidade de $80 \%$ da capacidade de campo e profundidades de $0-30 \mathrm{~cm}(\mathrm{~A})$ e $50-70 \mathrm{~cm}(\mathrm{~B})$ 
de cinética de primeira ordem para descrever a degradação do endossulfam no solo e as condições estudadas. Todos os parâmetros estimados de ambos os modelos testados, foram estatisticamente significativos pelo teste $\mathrm{t}(\mathrm{P}<0,05)$ conforme Tabelas 1 e 2 , além de mais de $90 \%$ dos resíduos padronizados se situarem no intervalo entre 2 e -2 .

Tabela 1. Parâmetros do modelo de cinética de primeira ordem para degradação do endossulfam em um Latossolo Vermelho distrófico em Ponta Porã, MS

\begin{tabular}{|c|c|c|c|c|c|}
\hline \multirow[b]{2}{*}{$\begin{array}{l}\text { Prof. } \\
\text { (cm) }\end{array}$} & \multicolumn{5}{|c|}{ Cinética de primeira ordem } \\
\hline & $M_{0}(\%)$ & $k\left(d^{-1}\right)$ & & $\mathrm{TD}_{90}$ & $\begin{array}{c}\text { Erro } \chi^{2} \\
(\%)\end{array}$ \\
\hline $\begin{array}{r}0-30 \\
50-70\end{array}$ & $\begin{array}{l}89,69 \pm 2,26 \\
84,70 \pm 2,37^{*}\end{array}$ & $\begin{array}{l}0,035 \pm 0,002^{*} \\
0,027 \pm 0,002^{*}\end{array}$ & $\begin{array}{l}20 \\
26\end{array}$ & $\begin{array}{l}65 \\
86\end{array}$ & $\begin{array}{l}6,3 \\
7,6\end{array}$ \\
\hline
\end{tabular}

*Significativo pelo teste t a 5\%; $\mathrm{TD}_{50}$ - tempo para degradação de $50 \%$ da dose inicial aplicada; $\mathrm{TD}_{90}$ - tempo para degradação de $90 \%$ da dose inicial aplicada

Tabela 2. Parâmetros do modelo bifásico de Gustafson \& Holden (1990) para degradação do endossulfam em um Latossolo Vermelho distrófico em Ponta Porã, MS

\begin{tabular}{|c|c|c|c|c|c|c|}
\hline \multirow[b]{2}{*}{$\begin{array}{l}\text { Prof. } \\
\text { (cm) }\end{array}$} & \multicolumn{6}{|c|}{ Bifásico - Gustafson \& Holden (1990) } \\
\hline & $M_{0}(\%)$ & $\alpha(-)$ & $\beta$ (dia) & $\frac{T_{50}}{(d}$ & $\frac{T D_{90}}{a s)}$ & $\begin{array}{c}\text { Erro } \chi^{2} \\
(\%)\end{array}$ \\
\hline 0 & $0,1-2$ & $1,17 \pm 0,29^{*}$ & $0,05 \pm 0,02^{*}$ & 17 & 132 & 2,6 \\
\hline $50-70$ & $89,03 \pm 2,69^{*}$ & $1,46 \pm 0,47^{\star}$ & $0,03 \pm 0,01^{*}$ & 23 & 143 & 7,0 \\
\hline
\end{tabular}

${ }^{*}$ Significativo pelo teste t a $5 \%$; $\mathrm{TD}_{50}$ - tempo para degradação de $50 \%$ da dose inicial aplicada; $\mathrm{TD}_{90}$ - tempo para degradação de $90 \%$ da dose inicial aplicada

Os valores de $\mathrm{TD}_{50}$ do endossulfam estimados com base no modelo de cinética de primeira ordem, foram de 20 dias para 0-30 $\mathrm{cm}$ e 26 dias para $50-70 \mathrm{~cm}$ de profundidade (Tabela 1). Esses valores são inferiores aos reportados por PPDB (2011) que variaram entre 28 e 50 dias para solos incubados a 20 ${ }^{\circ} \mathrm{C}$. Como neste estudo os solos foram incubados a $30^{\circ} \mathrm{C}$, os menores valores de $\mathrm{TD}_{50}$ se deveram, quando comparados com os de PPDB (2011) possivelmente a uma degradação mais rápida devido à temperatura mais elevada. Já os valores de $\mathrm{TD}_{50}$ estimados com base no modelo bifásico foram de 17 dias para 0-30 $\mathrm{cm}$ e 23 dias para $50-70 \mathrm{~cm}$ de profundidade (Tabela 2). De maneira geral, os valores de $\mathrm{TD}_{50}$ estimados com base no modelo bifásico foram inferiores aos obtidos pelo modelo de cinética de primeira ordem, evidenciando a necessidade da escolha correta e com base em critérios estatísticos de um modelo de degradação para estimativa dos valores de $\mathrm{TD}_{50}$. Com base no modelo de cinética de primeira ordem, as estimativas de $\mathrm{TD}_{90}$ foram de 65 dias para $0-30 \mathrm{~cm}$ e 86 dias para $50-70 \mathrm{~cm}$ de profundidade (Tabela 1); apesar disto, esses valores foram bem superiores quando estimados pelo modelo bifásico e iguais a 132 dias para $0-30 \mathrm{~cm}$ e 143 dias para $50-70 \mathrm{~cm}$ de profundidade (Tabela 2). Observa-se que houve tendência do modelo bifásico estimar valores superiores da quantidade remanescente de endossulfam para ambas as profundidades quando comparado ao modelo de cinética de primeira ordem, para as últimas datas de amostragem (Figura 1). Com isto, são esperados valores superiores de $\mathrm{TD}_{90}$ para o modelo bifásico devido à menor taxa de diminuição das concentrações remanescentes de endossulfam para este modelo para tempo superiores a 50 dias. Salienta-se que estimativas de $\mathrm{TD}_{90}$ em experimentos de degradação em que a concentração do agrotóxico na última data de amostragem foi superior a $10 \%$ da dose inicial aplicada, devem ser interpretadas com cautela já que uma predição de valores por modelos matemáticos fora do intervalo estudado pode ser perigosa.

A conversão de endossulfam em sulfato de endossulfam foi mais rápida na profundidade de $0-30 \mathrm{~cm}$ quando comparada a 50-70 cm de profundidade, sendo as maiores concentrações observadas aos 43 dias após aplicação do endossulfam para ambas as profundidades (Figura 2). Os ajustes das concentrações de sulfato de endossulfam em função do tempo para ambas as profundidades, ao modelo de cinética de primeira ordem, resultaram em valores de Erro $\chi^{2}$ iguais a 15,4 para 0-30 $\mathrm{cm}$ e 17,7 para 50-70 $\mathrm{cm}$ de profundidade (Tabela 3 ). Os

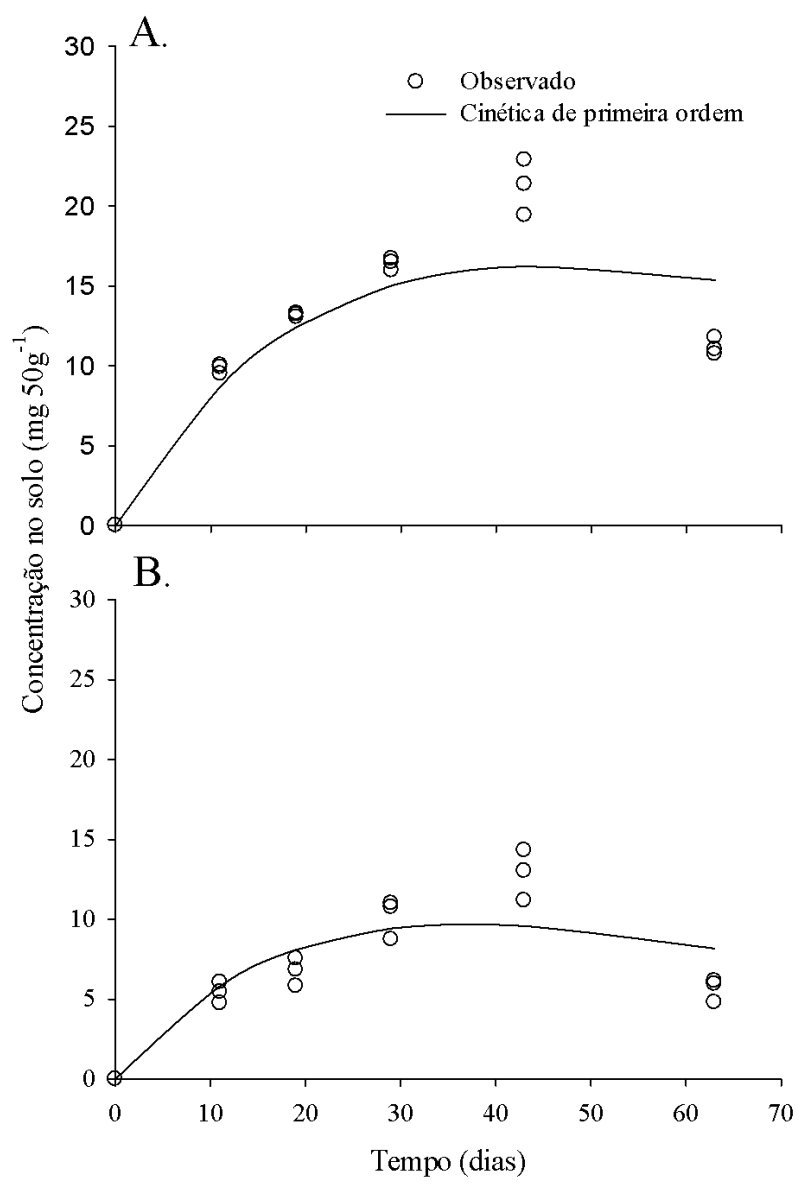

Figura 2. Cinética de formação e degradação do metabólito sulfato de endossulfam em um Latossolo Vermelho distrófico incubado a $30{ }^{\circ} \mathrm{C}$, umidade de $80 \%$ da capacidade de campo e profundidades de 0-30 cm (A) e 50-70 cm (B)

Tabela 3. Parâmetros do modelo de cinética de primeira ordem para cinética de formação e degradação do sulfato de endossulfam em um Latossolo Vermelho distrófico em Ponta Porã, MS.

\begin{tabular}{|c|c|c|c|c|}
\hline \multirow{3}{*}{$\begin{array}{l}\text { Prof. } \\
\text { (cm) }\end{array}$} & \multicolumn{4}{|c|}{ Cinética de Primeira Ordem } \\
\hline & \multirow{2}{*}{$\begin{array}{c}k_{\text {sul }} \\
\left(d^{-1}\right)\end{array}$} & $\mathrm{TD}_{50}$ & $\mathrm{TD}_{90}$ & \multirow{2}{*}{$\begin{array}{c}\text { Erro } \chi^{2} \\
(\%)\end{array}$} \\
\hline & & \multicolumn{2}{|c|}{ (dias) } & \\
\hline $0-30$ & $0,012 \pm 0,002^{*}$ & 59 & 195 & 15,4 \\
\hline $50-70$ & $0,026 \pm 0,004^{*}$ & 26 & 87 & 17,7 \\
\hline
\end{tabular}

${ }^{*}$ Significativo pelo teste t a 5\%; TD50 - tempo para degradação de $50 \%$ da dose inicial aplicada TD90 - tempo para degradação de $90 \%$ da dose inicial aplicada 
valores de $\mathrm{k}_{\text {sul }}$ para as duas profundidades foram estatisticamente significativos pelo teste $\mathrm{t}(\mathrm{P}<0,05)$, conforme a Tabela 3 , além de mais de $90 \%$ dos resíduos padronizados se situarem no intervalo entre 2 e -2 . Os valores de $\mathrm{TD}_{50}$ e $\mathrm{TD}_{90}$ para o sulfato de endossulfam foram, respectivamente, de 59 e 195 dias para $0-30 \mathrm{~cm}$ e 26 e 87 dias para $50-70 \mathrm{~cm}$ de profundidade (Tabela 3). Assim, observa-se uma degradação mais rápida do metabólito sulfato de endossulfam na camada mais profunda do solo (50-70 cm). Silva et al. (2010) avaliaram a influência da umidade, temperatura e profundidade do solo na degradação do sulfato de endossulfam em solos incubados provenientes de uma região agrícola de Mato Grosso e observaram valores de $\mathrm{TD}_{50}$ entre 6 a 88 dias. A degradação mais rápida de sulfato de endossulfam na camada mais profunda do solo, conforme constatado neste estudo, também foi observada por Silva et al. (2010). É provável que esta degradação mais rápida na camada mais profunda do solo tenha sido favorecida pelo maior teor de argila quando comparada com a superfície, o que pode favorecer o processo denominado hidrólise catalisada pela argila (Awasthi \& Prakash, 1997).

\section{ConClusões}

1. No campo, o endossulfam apresentou baixa lixiviação com profundidade máxima de $10 \mathrm{~cm}$ e uma baixa persistência para as condições edafoclimáticas estudadas.

2. Os valores de $\mathrm{TD}_{50}$ para o endossulfam nas duas profundidades estudadas, variaram de 17 a 26 dias e para o sulfato de endossulfam, de 26 a 59 dias.

3. O modelo bifásico de degradação mostrou-se superior para descrever os dados de degradação de endossulfam em laboratório, quando comparado ao de primeira ordem.

\section{Agradecimentos}

Ao CNPq, pelo auxílio financeiro (processos $n^{\circ} 471517 / 2007$ 0 e $n^{\circ} 551696 / 2007-9$ ).

\section{Literatura Citada}

Aldworth, J.; Jackson, S. H. Statistical tools for determining appropriate selection of regression models for analysis of environmental fate datasets. Pest and Management Science, v.64, p.536-543, 2008.

ANVISA - Agência Nacional de Vigilância Sanitária. Resolução-RDC n 28, de 9 de agosto de 2010: Regulamento técnico para o ingrediente ativo Endossulfam em decorrêcia da reavaliação toxicológica. Brasília, DF, 2010. <http:// www.brasilsus.com.br/legislacoes/resolucoes/105067-28. html>. 14 Mai. 2012.

Awasthi, M. D.; Prakash, N. B. Persistence of chlorpyrifos in soils under different moisture regimes. Pesticide Science, v.50, p.1-4, 1997.

Corrêa, C. M. D.; Oliveira, J. J. V.; Tornisielo, V. L. Resíduos de endosulfan em solo (Latossolo Roxo) cultivado com soja [Glycinemax (L.) Merrill]. Revista do Instituto Adolfo Lutz, v.62, p.69-75, 2003.
Dubus, I. G.; Hollis, J. M.; Brown, C. D. Pesticides in rainfall in Europe. Environmental Pollution, v.110, p.331-344, 2000.

Gish, T. J.; Prueger, J. H.; Daughtry, C. S. T.; Kustas, W. P.; McKee, L. G.; Russ, A. L.; Hatfield, J. L. Comparison of field-scale herbicide runoff and volatilization losses: an eight-year field investigation. Journal of Environmental Quality, v.40, p.1432-1442, 2011.

Guidance for evaluating laboratory and field dissipation studies to obtain DegT50 values of plant protection products in soil. European Food Safety Authority Journal, v.8, p.1-67, 2010.

Gustafson, D.; Holden, L. R. Nonlinear pesticide dissipation in soil: a new model based on spatial variability. Environmental Science and Technology, v.24, p.1023-1038, 1990.

Kennedy, I. R.; Sánchez-Bayo, F.; Kimber, S. W.; Hugo, L.; Ahmad, N. Off-site movement of endosulfan from irrigated cotton in New South Wales. Journal of Environmental Quality, v.30, p.683-696, 2001.

Köhne, J. M.; Köhne, S.; Simunek, J. A review of model applications for structured soils: b) pesticide transport. Journal of Contaminant Hydrology, v.104, p.36-60, 2009.

Laabs, V.; Amelung, W.; Pinto, A.; Altstaedt, A.; Zech, W. Leaching and degradation of corn and soybean pesticides in an Oxisol of the Brazilian Cerrados. Chemosphere, v.41, p.1441-1449, 2000.

Laabs, V.; Amelung, W.; Pinto, A.; Wantzen, M.; Silva, C. J.; Zech, W. Pesticides in surface water, sediment, and rainfall of the northeastern Pantanal Basin, Brazil. Journal of Environmental Quality, v.31, p.1636-1648, 2002.

Leonard, A. W.; Hyne, R. V.; Lim, R. P.; Leigh, K. A.; Le, J.; Beckett, R. Fate and toxicity of endosulfan in Namoi River water and bottom sediment. Journal of Environmental Quality, v.30, p.750-759, 2001.

Meyer, H. KinGUI: Manual for the graphical user interface: manual version: 0.16, KinGII version: 2.2012.202, KinGII Viewer version: 2.2012.203. [S.1.]: Bayer CropScience AG, 2012. n.p.

Neter, J.; Kutner, M. H.; Nachtsheim, C. J.; Wasserman, W. Applied linear statistical models. 4.ed. Chicago: Irwin, 1996. 1408p.

Nogueira, E. N.; Dores, E. F. G. C.; Pinto, A. A.; Amorim, R. S. S.; Ribeiro, M. L.; Lourencetti, C. Currently used pesticides in water matrices in Central-Western Brazil. Journal of the Brazilian Chemical Society, v.23, p.1476-1487, 2012.

Oliveira, M. F.; Brighenti, A. M. Comportamento dos herbicidas no ambiente. In: Oliveira Júnior, R. S.; Constantin, J.; Inoue, M. H. Biologia e manejo de plantas daninhas. Curitiba: Omnipax, 2011. p.263-304.

Pignatello, J. J. The measurement and interpretation of sorption and desorption rates for organic compounds in soil media. Advances in Agronomy, v.69, p.1-73, 2000.

PPDB - Pesticide Properties Database. Hatfield: University of Hertfordshire: AERU , 2011.<http://sitem.herts.ac.uk/aeru/ footprint/index2.htm>. 14 Mai. 2012.

Richter, O.; Diekkrüger, B.; Nörtersheuser. Environmental fate and modelling of pesticides: from the laboratory to the field scale. Weinheim: VCH, 1996. 281p.

Santos, H. G.; Jacomine, P. K. T.; Anjos, L. H. C.; Oliveira, V. A.; Oliveira, V. B.; Coelho, M. R.; Lumbreas, J. F.; Cunha, T. J. F. Sistema brasileiro de classificação de solos. 2.ed. Rio de Janeiro: Embrapa Solos, 2006. 306p. 
Scheyer, A.; Graeff, C. C.; Morville, S.; Mirabel, P.; Millet, $\mathrm{M}$. Analysis of some organochlorine pesticides in an urban atmosphere (Strasbourg, east of France). Chemosphere, v.58, p.1517-1524, 2005.

Scorza Júnior, R. P.; Rigitano, R. L. O. Sorção, degradação e lixiviação do inseticida tiametoxam em dois solos de Mato Grosso do Sul. Revista Brasileira de Engenharia Agrícola e Ambiental, v.16, p.564-572, 2012.
Silva, F. A.; Lourencetti, C.; Dores, E. F. G. C. Influência da temperatura, umidade e profundidade do solo na persistência do diurom e sulfato de endossulfam em um solo tropical. Química Nova, v.33, p.1457-1463, 2010.

Vogel, J. R.; Majewski, M. S.; Capel, P. D. Pesticides in rain in four agricultural watersheds in the United States. Journal of Environmental Quality, v.37, p.1101-1115, 2008. 